\title{
Study on Fault Diagnosis Technologies of HVDC Light System
}

\author{
Yiming Jiang \\ School of Precision Instrument and Opto-electronics Engineering, Tianjin University, \\ Tianjin, 300072, China \\ jymyx@126.com
}

\begin{abstract}
Because of high reliability and other characteristics, High Voltage Direct Current (HVDC) Light system had important applications in power transmission system. In order to ensure its operation safety, the fault diagnosis technologies based on observer were researched. The model of HVDC light system was introduced, and the types and characteristics of the system were analyzed. Several common fault diagnosis methods were discusses, including method based on analytical model, method based on expert system and method based on neural network. A fault diagnosis observer was designed, the output waveforms of observer were observed and analyzed when the failure occurred, the type of fault was determined, and finally, the automatic detection of fault in HVDC system was achieved. The test simulation results showed that this method detected the occurrence of failures, identified the faults in characteristics and provided evidence for exclusion and maintenance of fault.
\end{abstract}

Keywords: HVDC light, fault diagnosis, observer, analytical model

\section{Introduction}

Under certain conditions, High Voltage Direct Current transmission, HVDC, has more advantages than alternating current transmission and it is especially suitable for long distance and large capacity transmission. The traditional HVDC uses line commutated current source converter based on grid-based thyristor switch. The HVDC Light makes the voltage source converter as core, using turn-off devices such as insulated gate bipolar transistor in hardware and pulse width modulation technique in controlling so as to achieve the purpose of high and controllable direct current transmission.

With its unique advantages, HVDC Light will have a place in the next power transmission system. HVDC systems are often used as the links between major grids. If the system has malfunction and interruption of power supply, it will enable suspension of production so as to cause confusion, and may even endanger the safety of people and equipments. Then, the fault diagnosis is the key factor of HVDC technology, and is the assurance for the reliability of power supply of HVDC system $[1,2]$.

The failure of power system may be caused by short circuit, open circuit of some sections or functional failure if parts of components of the system. When system failure occurs, all or part of the system parameter variables have different characteristics with the normal states, this difference can contain a wealth of fault information. The task of fault diagnosis is to describe the characteristics of system failure and use the description to detect and isolate these system failures.

This study adopts the method of fault diagnosis observer to research the fault diagnosis of HVDC Light system. Firstly, fault diagnosis observer is built, and then the residual system is 
established according to the state equations of HVDC light system, finally the HVDC Light system fault diagnosis is studied based on fault diagnosis observer.

\section{HVDC System Model}

\subsection{Modeling of HVDC System}

HVDC Light system consists of two pulse width modulated voltage source inverters $\left({ }^{V S C_{1}}\right.$, $\left.V S C_{2}\right)$, two DC capacitors $\left(C_{1}, C_{2}\right)$, two commutation reactors $\left(L_{1}, L_{2}\right)$ and DC transmission lines, usually, $L_{1}=L_{2}=L, C_{1}=C_{2}=C$. Where, $C$ is to provide voltage supporting and reduce the harmonics on DC side, $L$ is used to filter the output current harmonics. The losses of current converter and its line are expressed with resistors $R_{1}=R_{2}=R$, the loss of DC line is resistor $R_{d}$ [3]. Discarding $R L C$ branches and amplifying rectification, inverter and three-phase branches, the system can be simplified, and the basic structure of HVDC Light is obtained, as shown in Figure 1.

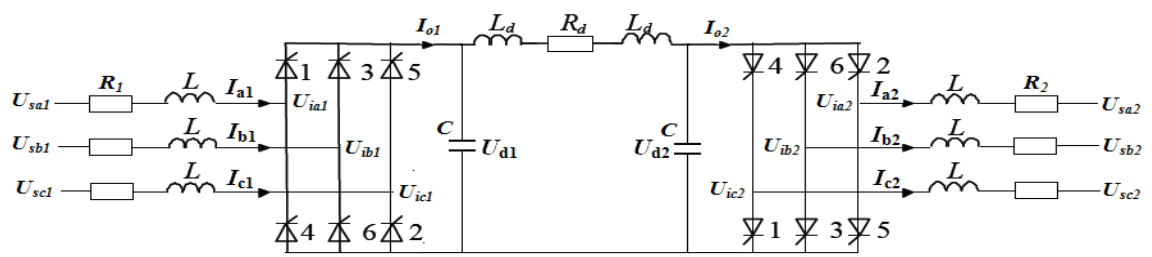

Figure 1. The Simplified Structure of HVDC Light System

In order to facilitate the system analysis, according to the system model, when ignoring the inductance on the line, the dynamic differential equations of three-phase stationary coordinate system in AC side can be gotten [4], as shown in equation (1).

$$
\begin{cases}L \frac{\mathrm{d} I_{a 1}}{\mathrm{~d} t}+R I_{a 1}=U_{s a 1}-U_{i a 1} & \left\{\begin{array}{l}
\mathrm{d} I_{a 2} \\
\mathrm{~d} t
\end{array} \quad R I_{a 2}=U_{i a 2}-U_{s a 2}\right. \\
L \frac{\mathrm{d} I_{b 1}}{\mathrm{~d} t}+R I_{b 1}=U_{s b 1}-U_{i b 1} & \left\{\frac{\mathrm{d} I_{b 2}}{\mathrm{~d} t}+R I_{b 2}=U_{i b 2}-U_{s b 2}\right. \\
L \frac{\mathrm{d} I_{c 1}}{\mathrm{~d} t}+R I_{c 1}=U_{s c 1}-U_{i c 1} & L \frac{\mathrm{d} I_{c 2}}{\mathrm{~d} t}+R I_{c 2}=U_{i c 2}-U_{s c 2}\end{cases}
$$

where, $I_{a 1}$ and $I_{a 2}$ are the currents of phase $A ; U_{s a 1}$ and $U_{s a 2}$ are the voltages of phase $A$ in AC systems 1 and 2. $U_{i a 1}$ and $U_{i a 2}$ are the voltages of phase $A$ in AC side. Similarly, variables of phases $B$ and $C$ can be defined as equation (1).

According to the low of power balance, the expression can be gotten as equation (2).

$$
\left\{\begin{array}{l}
P_{c 1}=U_{i a 1} I_{a 1}+U_{i b 1} I_{b 1}+U_{i c 1} I_{c 1}=U_{d 1} I_{01} \\
P_{c 2}=U_{i a 2} I_{a 2}+U_{i b 2} I_{b 2}+U_{i c 2} I_{c 2}=U_{d 2} I_{02}
\end{array}\right.
$$

where, $P_{c 1}$ and $P_{c 2}$ are respectively the input and output active power in $\mathrm{AC}$ sides of $V S C_{1}$, $V S C_{2} ; U_{d 1}$ and $U_{d 2}$ are voltages in DC side; $I_{01}, I_{02}$ are respectively the input and output current in DC side. 
Supposing that the amplitude of input voltage on the left side is $U_{s 1}$, and that of output voltage on right side is $U_{s 2}$, then, the relation can be gotten as shown in equation (3).

$\left\{\begin{array}{l}U_{s a 1}=U_{s 1} \cos \omega_{1} t \\ U_{s b 1}=U_{s 1} \cos \left(\omega_{1} t-2 \pi / 3\right) \\ U_{s c 1}=U_{s 1} \cos \left(\omega_{1} t+2 \pi / 3\right)\end{array} \quad\left\{\begin{array}{l}U_{s a 2}=U_{s 2} \cos \omega_{2} t \\ U_{s b 2}=U_{s 2} \cos \left(\omega_{2} t-2 \pi / 3\right) \\ U_{s c 2}=U_{s 2} \cos \left(\omega_{2} t+2 \pi / 3\right)\end{array}\right.\right.$

where, $\omega_{1}$ and $\omega_{2}$ are respectively the voltage angular frequency of AC systems 1 and 2 .

Additionally, it is can be gotten as equation (4).

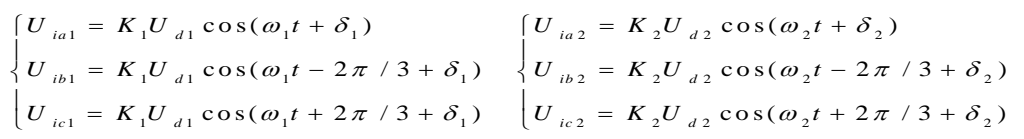

where, $K_{1}$ and $K_{2}$ are modulation ratios of $V S C_{1}$, and $V S C_{2}$ which values are equal to the ratios of phase voltage amplitude of AC fundamental of inverter output to the voltages in AC side. $\delta_{1}$ and $\delta_{2}$ are the phase angle differences between phase voltages of AC systems and AC fundamental phase voltages of output of $V S C_{1}$, and $V S C_{2}$.

Eliminating $U_{s a}, U_{s b}, U_{s c}$ and $U_{i a}, U_{i b}, U_{i c}$, equation (1) can be expressed as equation (5).

$\left\{\begin{array}{l}L \frac{\mathrm{d} I_{a 1}}{\mathrm{~d} t}+R I_{a 1}=U_{s 1} \cos \omega_{1} t-K_{1} U_{d 1} \cos \left(\omega_{1} t+\delta_{1}\right) \\ L \frac{\mathrm{d} I_{b 1}}{\mathrm{~d} t}+R I_{b 1}=U_{s 1} \cos \left(\omega_{1} t-2 \pi / 3\right)-K_{1} U_{d 1} \cos \left(\omega_{1} t-2 \pi / 3+\delta_{1}\right) \\ L \frac{\mathrm{d} I_{c 1}}{\mathrm{~d} t}+R I_{c 1}=U_{s 1} \cos \left(\omega_{1} t+2 \pi / 3\right)-K_{1} U_{d 1} \cos \left(\omega_{1} t+2 \pi / 3+\delta_{1}\right)\end{array}\right.$
$\left\{\begin{array}{l}L \frac{\mathrm{d} I_{a 2}}{\mathrm{~d} t}+R I_{a 2}=K_{2} U_{d 2} \cos \left(\omega_{2} t+\delta_{2}\right)-U_{s 2} \cos \omega_{2} t \\ L \frac{\mathrm{d} I_{b 2}}{\mathrm{~d} t}+R I_{b 2}=K_{2} U_{d 2} \cos \left(\omega_{2} t-2 \pi / 3+\delta_{2}\right)-U_{s 2} \cos \left(\omega_{2} t-2 \pi / 3\right) \\ \frac{\mathrm{d} I_{c 2}}{\mathrm{~d} t}+R I_{c 2}=K_{2} U_{d 2} \cos \left(\omega_{2} t+2 \pi / 3+\delta_{2}\right)-U_{s 2} \cos \left(\omega_{2} t+2 \pi / 3\right)\end{array}\right.$

In addition, the voltage dynamic equation in DC side can be gotten as equation (6).

$\left\{\begin{array}{l}C \frac{\mathrm{d} U_{d 1}}{\mathrm{~d} t}=I_{01}-I_{d} \\ C \frac{\mathrm{d} U_{d 2}}{\mathrm{~d} t}=I_{d}-I_{02}\end{array}\right.$

where, $I_{01}$ and $I_{02}$ are respectively DC currents in rectifier and inverter, which can be gotten as equation (7).

$\left\{\begin{array}{l}I_{01}=K_{1}\left[\cos \left(\omega_{1} t+\delta_{1}\right) I_{a 1}+\cos \left(\omega_{1} t-2 \pi / 3+\delta_{1}\right) I_{b 1}+\cos \left(\omega_{1} t+2 \pi / 3+\delta_{1}\right) I_{c 1}\right] \\ I_{02}=K_{2}\left[\cos \left(\omega_{2} t+\delta_{2}\right) I_{a 2}+\cos \left(\omega_{2} t-2 \pi / 3+\delta_{2}\right) I_{b 2}+\cos \left(\omega_{2} t+2 \pi / 3+\delta_{2}\right) I_{c 2}\right]\end{array}\right.$

Substituting $I_{01}$ and $I_{02}$ to equation (6) to get a new expression as equation (8). 


$$
\left\{\begin{array}{l}
C \frac{\mathrm{d} U_{d 1}}{\mathrm{~d} t}=K_{1}\left[\cos \left(\omega_{1} t+\delta_{1}\right) I_{a 1}+\cos \left(\omega_{1} t-2 \pi / 3+\delta_{1}\right) I_{b 1}+\cos \left(\omega_{1} t+2 \pi / 3+\delta_{1}\right) I_{c 1}\right]-I_{d} \\
C \frac{\mathrm{d} U_{d 2}}{\mathrm{~d} t}=I_{d}-K_{2}\left[\cos \left(\omega_{2} t+\delta_{2}\right) I_{a 2}+\cos \left(\omega_{2} t-2 \pi / 3+\delta_{2}\right) I_{b 2}+\cos \left(\omega_{2} t+2 \pi / 3+\delta_{2}\right) I_{c 2}\right]
\end{array}\right.
$$

\subsection{State Equations of HVDC Light Mathematic Model}

From the equations above, the mathematical model can be modified into two state equations of rectifier and inverter as equation (9) and equation (10).
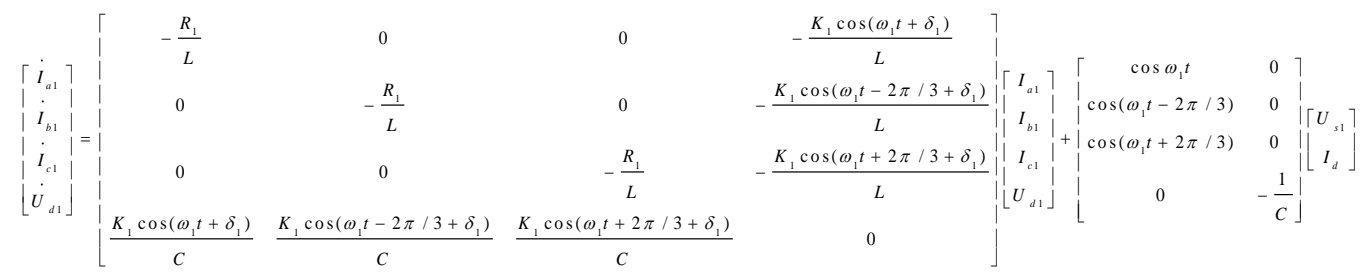

where, $\left[\begin{array}{llll}\dot{I}_{a 1} & \dot{I}_{b 1} & \dot{I}_{c 1} & \dot{U}_{d 1}\end{array}\right]$ is the state in rectifier side, $k_{1}$ is the modulation ration of $V S C_{1}$.

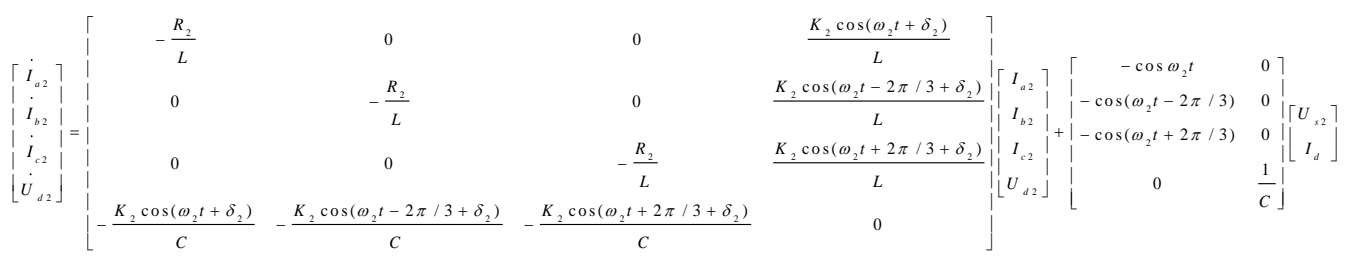

where, $\left[\begin{array}{llll}\dot{I}_{a 2} & \dot{I}_{b 2} & \dot{I}_{c 2} & \dot{U}_{d^{2}}\end{array}\right]$ is the state of inverter side, $k_{2}$ is the modulation ration of $V S C_{2}$.

Analyzing the DC cable s of HVDC Light system, $I_{d}$ has the relation as shown in equation (11).

$L_{d} \frac{\mathrm{d} I_{d}}{\mathrm{~d} t}=U_{d 1}-U_{d 2}-I_{d} R_{d}$

Ignoring the line inductance $L_{d}$, it can be changed as equation (12).

$I_{d}=\frac{U_{d 1}-U_{d 2}}{R_{d}}$

The states can be simplified as equation (13).

$\left[\begin{array}{l}\dot{U}_{d 1} \\ \dot{U}_{d 2}\end{array}\right]=\left[\begin{array}{ll}-\frac{1}{R_{d} C} & \frac{1}{R_{d} C} \\ \frac{1}{R_{d} C} & -\frac{1}{R_{d} C}\end{array}\right]\left[\begin{array}{l}U_{d 1} \\ U_{d 2}\end{array}\right]+\left[\begin{array}{c}\frac{K_{1} \cos \left(\omega_{1} t+\delta_{1}\right)}{C} I_{a 1}+\frac{K_{1} \cos \left(\omega_{1} t-2 \pi / 3+\delta_{1}\right)}{C} I_{b 1}+\frac{K_{1} \cos \left(\omega_{1} t+2 \pi / 3+\delta_{1}\right)}{C} I_{c 1} \\ C\end{array}\right]$

Under ordinary conditions, $R_{1}=R_{2}=R$, it can be gotten as equations (14) and (15). 


$$
\begin{aligned}
& \dot{x}=A(t) x+\left[\begin{array}{cc}
\cos \omega_{1} t & 0 \\
\cos \left(\omega_{1} t-2 \pi / 3\right) & 0 \\
\cos \left(\omega_{1} t+2 \pi / 3\right) & 0 \\
0 & \cos \omega_{2} t \\
0 & \cos \left(\omega_{2} t-2 \pi / 3\right) \\
\mid & \cos \left(\omega_{2} t+2 \pi / 3\right) \mid \\
\mid & 0
\end{array}\right] \\
& {\left[\begin{array}{l}
y_{1} \\
y_{2}
\end{array}\right]=\left[\begin{array}{cccccccc}
0 & 0 & 0 & 0 & 0 & 0 & 1 & 0 \\
0 & 0 & 0 & 0 & 0 & 0 & \frac{1}{R_{d} C} & -\frac{1}{R_{d} C}
\end{array}\right]^{x}}
\end{aligned}
$$

To facilitate the expression, the matrix $A(t)$ is divided into blocks in accordance with the state quantities $I$ and $U$, namely, it is expressed as equation (16) .

$$
A(t)=\left[\begin{array}{cc}
\mathrm{II} & \mathrm{IU} \\
\mathrm{U} \mathrm{I} & \mathrm{U} \mathrm{U}
\end{array}\right]
$$

where, $I I, I U, U I, U U$ are blocking matrixes, they are respectively $6 \times 6,6 \times 2,2 \times 6$ and $2 \times 2$, their concrete forms are as equations (17) - (20).

$$
\mathrm{II}=-\frac{R}{L} \cdot \mathrm{E}
$$

where, $\mathrm{E}$ is the identity matrix of order six.

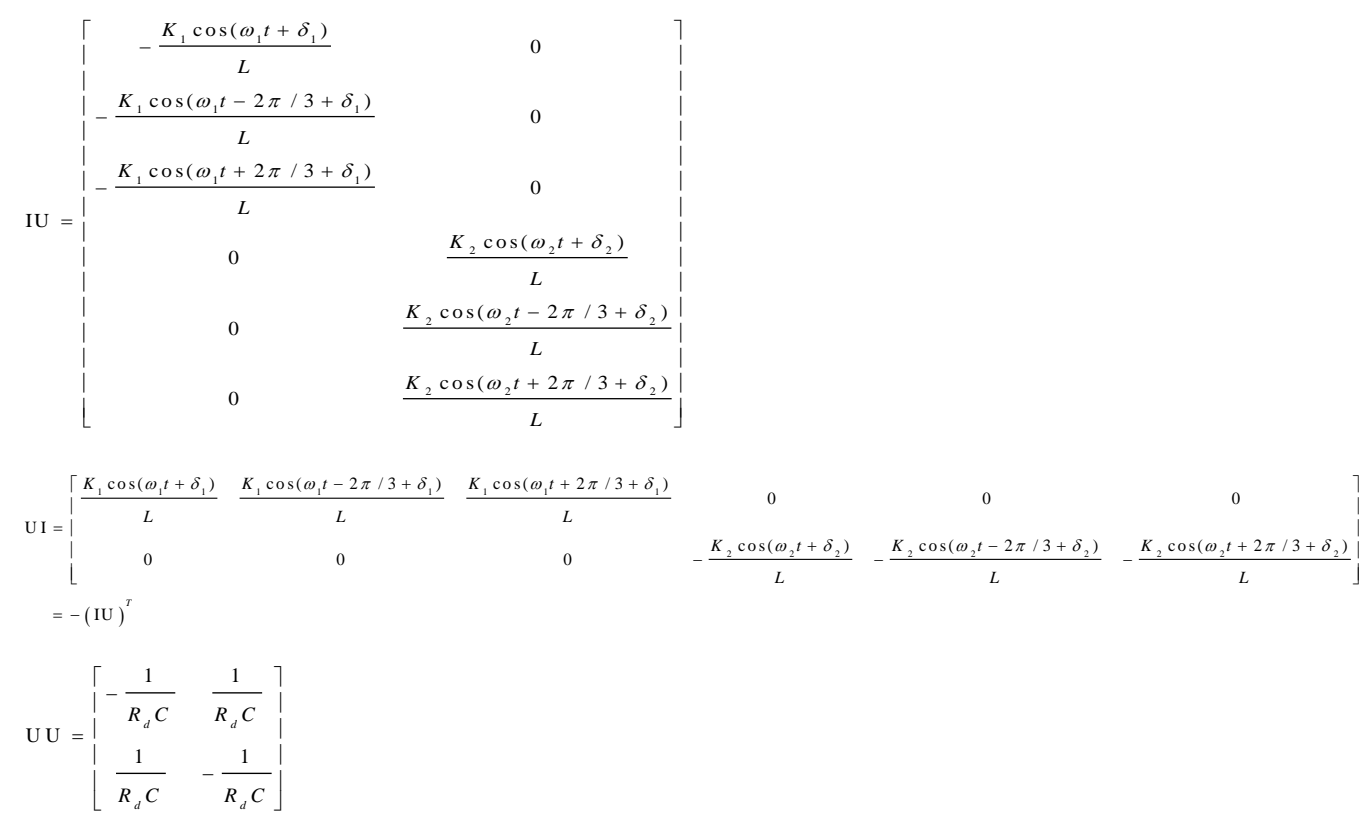

where,

$$
\begin{aligned}
& x=\left[\begin{array}{llllllll}
I_{a 1} & I_{b 1} & I_{c 1} & I_{a 2} & I_{b 2} & I_{c 2} & U_{d 1} & U_{d 2}
\end{array}\right]^{T} \\
& y=\left[\begin{array}{ll}
U_{d} & I_{d}
\end{array}\right]^{T}
\end{aligned}
$$


Blocking the matrix $A(t)$ can make the relation among $I_{a}, I_{b}, I_{c}$ and $U_{d}$ clear. The establishment of state equation is the basis of analyzing state observer.

\section{Fault and Diagnostic Methods of HVDC Light System}

Fault diagnosis is the core of DC control and protection system. In the DC system, the failure of any part can affect the safety and reliability of entire HVDC system. Therefore, fault diagnosis is the key factor of HVDC and the guarantee of power supply reliability.

\subsection{Failure Types}

The common faults of HVDC systems have the following main categories: single-phase short circuit of AC system sending end, three-phase short circuit of AC system sending end, open circuit of DC cable, monopole grounding of DC cable, dual pole grounding of DC cable, single-phase short circuit of AC system accepting end, three-phase short circuit of AC system accepting end, and so on.

For the HVDC system, the failures occur mainly in the DC area of converter station, overhead lines, cables and AC area of converter station. In general, the various types of failures are indicated in Table 1.

Table 1. The Types and Characteristics of Common Failures of HVDC System

\begin{tabular}{|l|l|}
\hline \multicolumn{1}{|c|}{ Failure types } & \multicolumn{1}{c|}{ Failure Characteristics } \\
\hline $\begin{array}{l}\text { Bridge short circuit of } \\
\text { rectification side }\end{array}$ & AC current increases and DC current drops \\
\hline $\begin{array}{l}\text { commutation failure of } \\
\text { inverter side }\end{array}$ & DC voltage drops and dc current increases \\
\hline DC transmission line fault & DC voltage steeply falls and DC current Steeply rises \\
\hline $\begin{array}{l}\text { AC system failure of } \\
\text { rectifier side }\end{array}$ & $\begin{array}{l}\text { AC voltage drops, DC voltage and current may correspondingly } \\
\text { decline, larger non-characteristic harmonics occur in unsymmetrical } \\
\text { fault }\end{array}$ \\
\hline $\begin{array}{l}\text { AC system failure of inverter } \\
\text { side }\end{array}$ & $\begin{array}{l}\text { Ac voltage drops, commutation failure may occur, larger non- } \\
\text { characteristic harmonics occur in unsymmetrical fault }\end{array}$ \\
\hline
\end{tabular}

\subsubsection{Line Failure}

The DC line failures are mostly pole-to-ground faults which cut off the power transmission on that electrode but the rest poles are actually not affected. The DC line faults have less destructive impact on the AC system connected to this line than those of AC system. Short circuit will transiently increase the rectifier currents and decrease the inverter currents.

The current control of rectifier is to reduce the DC voltage and return the current to its normal setting value $I_{0}$, the current of the inverter becomes smaller than the reference setting value $\left(I_{o}-I_{m}\right)$ of current controller. As a result, the operation mode of inverter control is changed from CEA control to CC control. This change lets the inverter voltage reduce to zero and changes its polarity. The voltage is equal to the RI voltage drop from each inverter to failed line. The rectifier current is $I_{o}$, but the current of inverter is $\left(I_{o}-I_{m}\right)$ in the opposite direction. Therefore, the steady-state fault current is equal to the current margin $I_{m}$, which is only $25 \%$ of the rated current [5]. 


\subsubsection{AC System Failure in Rectifier Side}

For the remote three-phase fault, the commutating voltage of rectifier side decreases very little which causes the decrease of rectifier current and then the drop of current. Current regulator reduces the angle $\alpha$ of inverter, and restores the current by increasing voltage. When $\alpha$ reaches the minimum threshold, the rectifier converts to the CIA control mode. This transfers the current control to the inverter which number of current commands is equal to the number of current commands of rectifier subtracting current margin.

If the low voltage continues, in order to restore the DC voltage and current to normal values, the transformer tap will act. According to number of voltage dropping, VDCOL can adjust the current and power transmission. For the adjacent three-phase fault, the commutating voltage of rectifier decreases significantly until the system is locked out under the control of VDCOL and the fault is eliminated.

Theoretically, the DC power can be transmitted at very low voltage of rectifier, which requires that the inverter reduces its voltage and greatly increases the angle $\beta$ to set the mode of current control. Ultimately, the impact increase of reactive power consumption on the AC system performance is more unfavorable than the DC system is locked out simply.

\subsubsection{AC System Failure in Inverter Side}

The remote three-phase fault can lead to the decreasing of inverter voltage and increasing of the DC current. The CC control of rectifier and CEA control of inverter respond to this change. If the low $\mathrm{AC}$ voltage continues, the transformer tap will change to recover the firing angle of the inverter and the DC voltage.

If the voltage drops significantly, the reducing of commutating voltage may cause temporary commutating failure of inverter before any action of correction control. When the inverter is running at $\gamma=18^{\circ}$, the voltage drops from $10 \%$ to $15 \%$, it will probably result in commutating failure.

Then, reducing the DC voltage of rectifier can match with the dropping of inverter DC voltage which can transfer some power. But, the caused reactive power increasing may be necessary to reduce the DC current, and VDCOL will generate this current decrease. In the extreme case of low voltage, repeat commutating failure is inevitable. Therefore, it may be necessary to lock off or bypass converter valve until the AC voltage is restored.

\subsection{Fault Diagnosis Methods}

When the failure of ppower system occurs, all or parts of the parameters exhibit different features which contain certain fault information. Fault diagnosis includes fault feature extraction, fault valuation, fault decision, and other parts.

Generally, the fault diagnosis methods are divided into two categories: model-based and non-model-based. They have different research methods, with advantages and disadvantages. For the HVDC system, the common fault diagnosis methods are as following.

\subsubsection{Method based on Analytical Model}

This method is used in identification techniques of online system which can establish the mathematical model for the system in real time. When there is failure, the input-output relationship of system is changed which will be reflected in the mathematical model. The method is feasible for particular power system models. 


\subsubsection{Method Based on Expert System}

Expert system is an intelligent system which uses specialized knowledge of experts in the research field to reason and solve difficult and practical problems. As the traditional expert system has some shortcomings, such as knowledge acquisition is difficult, knowledge ken is narrow, intelligence level is low, and so on, and then, its development has been limited.

\subsubsection{Method based on Neural Network}

The neural network simulates the learning function of human brain. It can obtain learning ability through training, resolve some problems those are difficult to handle by mathematical models or rules. Because neural network has strong nonlinear mapping ability, robustness and fault tolerance, it shows a certain advantage in fault diagnosis.

Currently, for the method based on analytical model, the fault diagnosis technologies of dynamic system are focused on the linear systems. There is less research on nonlinear systems. Because the assumptions on model in theoretical studies are often not met the practical applications, it has brought difficulties to the practical application of fault diagnosis technology. The state observer of modern control theory is important in fault diagnosis.

\section{Realization of Fault Diagnosis of HVDC Light System}

The process of fault diagnosis based on observer method is as follows. Firstly, a fault diagnosis observer is designed, when the fault occurs, the output waveform of observer is observed and analyzed, the fault type is determined, lastly, the automatic detection of fault in HVDC Light power transmission system is realized.

\subsection{Design of Fault Diagnosis Observer}

To a general linear time varying system, the status equation is as equation (23).

$\left\{\begin{array}{l}\dot{x}(t)=A(t) x(t)+B(t) u(t) \\ y(t)=C(t) x(t)\end{array}\right.$

where, ${ }^{x(t), y(t), u(t)}$ are respectively state vector, measuring output vector and control input vector.

The observer of fault diagnosis can be constructed as equation (24).

$\left\{\begin{array}{l}\dot{\hat{x}}(t)=[A(t)-L C(t)] \hat{x}(t)+L y(t)+B(t) u(t) \\ \hat{y}(t)=C(t) \hat{x}(t) \\ r(t)=y(t)-\hat{y}(t)\end{array}\right.$

where, $\hat{x}(t)$ and $\hat{y}(t)$ are respectively the estimate values of $x(t)$ and $y(t), r=y-\hat{y}$ is the residual, $L$ is the gain matrix of observer.

Then, define them as equation (25).

$\{e(t)=x(t)-\hat{x}(t)$

$\{r(t)=y(t)-\hat{y}(t)$

The relation can be gotten as equation (26).

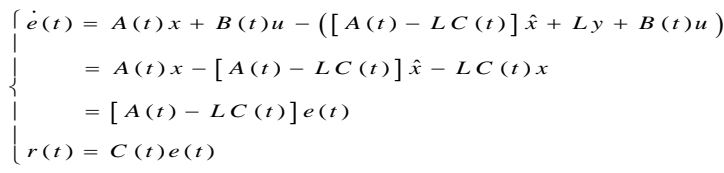


When $r \neq 0$, it indicates that the system has failures. Comparing different output waveforms of different faults can determine the fault type [6,7].

When fault occurs, taking into account the system noise, the state equation can be abbreviated to the nonlinear system model, as show in equation (27).

$\{\dot{x}(t)=A(t) x(t)+B(t) u(t)+f(t)+w(t)$

$\{y(t)=C(t) x(t)$

where, $f(t)$ is the fault vector; $w(t)$ is the Gaussian white noise which variance is $\sigma^{2}$ and mean is zero [8].

For example, while $U_{i a 2}$ and $U_{i b 2}$ are short-circuiting shown as Figure $1, U_{i a 2}$ and $U_{i b 2}$ change into equation (28).

$\left\{\begin{array}{l}U_{i a 2}=\frac{1}{2} K_{2} U_{d 2} \cos \left(\omega_{2} t-\pi / 3+\delta_{2}\right) \\ U_{i b 2}=\frac{1}{2} K_{2} U_{d 2} \cos \left(\omega_{2} t-\pi / 3+\delta_{2}\right)\end{array}\right.$

Thus, $A(t)$ Transforms into $\tilde{A}(t)$.

Accordingly, $f(t)$ could be expressed as equation (29).

$f(t)=[\tilde{A}(t)-A(t)] x(t)=\left[\begin{array}{cc}\mathrm{O} & \mathrm{F}_{2} \\ \mathrm{~F}_{1} & \mathrm{O}\end{array}\right]^{x(t)}$

where, $\mathrm{O}$ is Zero matrix, and $\mathrm{F}_{1}, \mathrm{~F}_{2}$ are shown as equation (30) according to equation (28).

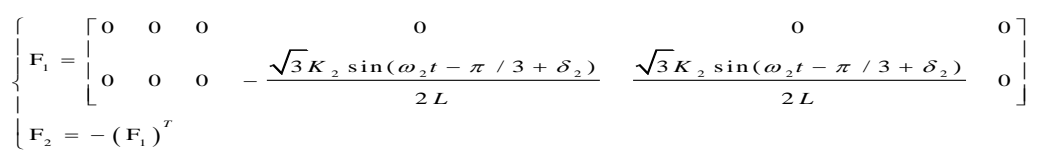

The observable matrix $Q$ of system is defined, as shown in equation (31).

$Q=\left[C_{0}^{\mathrm{T}}(t), C_{1}^{\mathrm{T}}(t), \ldots, C_{8}^{\mathrm{T}}(t)\right]^{\mathrm{T}}$

where,

$\left\{\begin{array}{l}C_{0}(t)=C(t) \\ C_{\mathrm{i}}(t)=\dot{C}_{\mathrm{i}-1}(t)+C_{\mathrm{i}-1}(t) A(t), i=1,2,3, \ldots, 7\end{array}\right.$

The constructed system is not completely observable. When some faults occur, the types can be determined, but which positions may not be found.

Since $f(t)$ is added to state equation, regardless of the noise. equation (33) is considered.

$\left\{\begin{array}{l}\dot{x}(t)=A(t) x(t)+B(t) u(t)+f(t) \\ \dot{\hat{x}}(t)=[A(t)-L C(t)] \hat{x}(t)+L y(t)+B(t) u(t)\end{array}\right.$

Equation (34) can be gotten by subtracting the above two equations.

$\dot{e}(t)=[A(t)-L C(t)] e(t)+f(t)$

When, the output is stable, that is, $\dot{e}(t)=0$, equation (35) can be gotten by transposing.

$[A(t)-L C(t)] r(t)=-C(t) f(t)$ 
Thus, $f(t)$ can be expressed by the change of $r(t)$.

\subsection{Output Simulation of Observer}

According to CIGRE model [9-11], supposing that, in the ideal situation, the HVDC system has a failure of the two-phase short circuit to ground, the faults simulation are studied by observing the output waves $U_{d}$ and $I_{d}$.

The output simulation waveforms are shown in Figure 2.

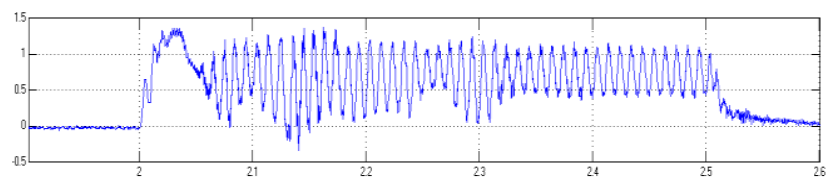

(a) single phase short circuit to ground

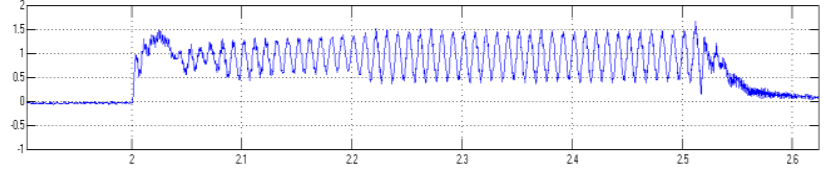

(b) two-phase short circuit to ground occurs

Figure 2. The Output of Observer to $U_{d}$

\subsection{Comparing and Analyzing of the Output Waveform of Observer}

The signal $U_{d}$ of observer vibrates acutely after rising. When it is stable, swing range is from about 0.4 to 1.5 which is periodic. The signal $I_{d}$ firstly drops below zero, and then increases in small oscillation, swinging range is from about 0.5 to 1.5 .

\subsection{Waveform Analysis of Fault}

From the above analysis, it can be seen that, for HVDC system, different types of faults have different output waveforms of $U_{d}$ and $I_{d}$ of corresponding state observer. So, the failures can be distinguished by different characteristics identification of their waveforms in principle.

Firstly, the stable waveforms of two-phase short circuit to ground are amplified. Taking the waveforms within $\Delta t=0.08 \mathrm{~s}$, the stable waveforms after failures occur show clear periodicity. The period is about $0.01 \mathrm{~s}$ which can be seen as the superposition of sine wave and disturbance wave. The two waves are as shown in Figure 3.

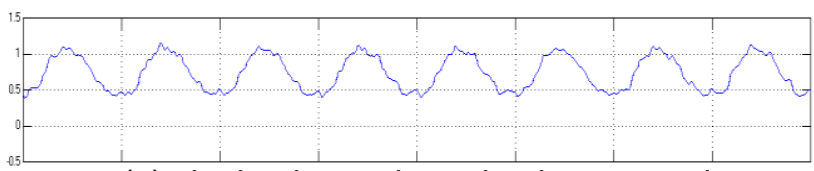

(a) single phase short circuit to ground

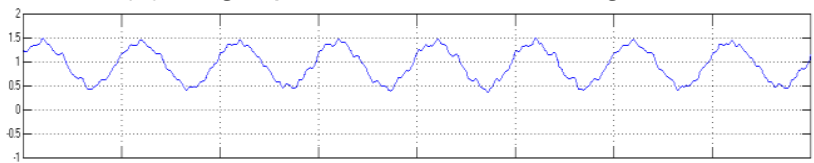

(b) two-phase short circuit to ground

Figure 3. Amplification of Stable Output of Observer to $U_{d}$ 
Since the output waveform has good periodicity, a stable rectangular wave can be obtained by using Schmitt trigger, and the noise and disturbance can be effectively filtered out. The output voltage of observer to Ud is connected to the Schmitt trigger circuit put voltage follower, setting that $\mathrm{VT}+=1$ and VT- $=0.5$, the adjustment process is shown in Figure 4.

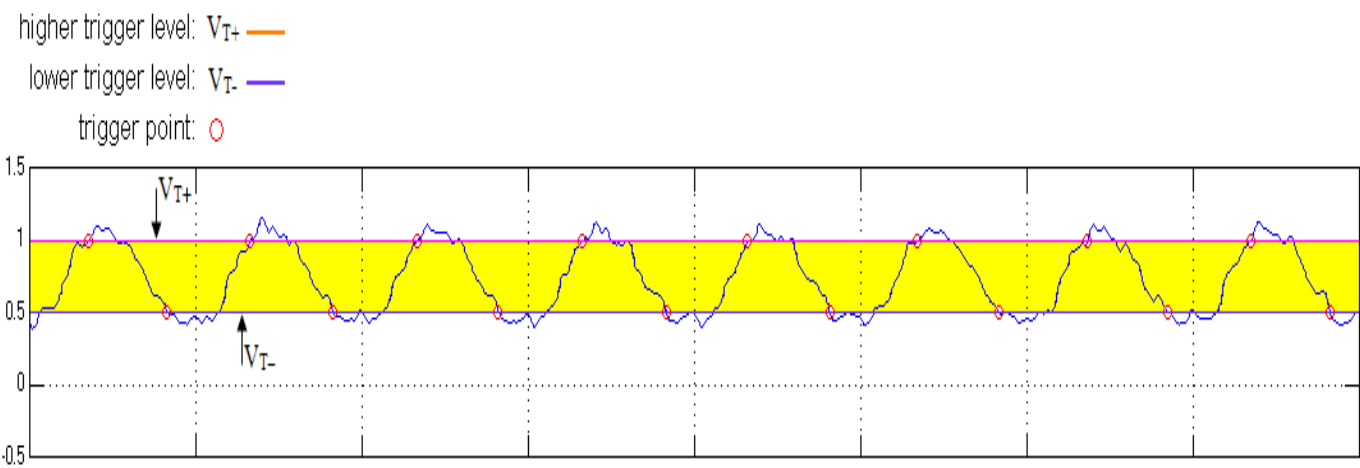

\section{Figure 4. The Adjustment Process of Outputting Voltage of Observer into Schmitt Trigger}

New waveform is obtained after this simulation, in order to compare it with the original waveform, the waveform within $\Delta t=0.08 \mathrm{~s}$ is still taken which is shown in Figure 5. Because of the memory performance and hysteresis of Schmitt trigger, at the same time, the noise and disturbance is removed, then, the period and duty cycle of the output square wave are stability.

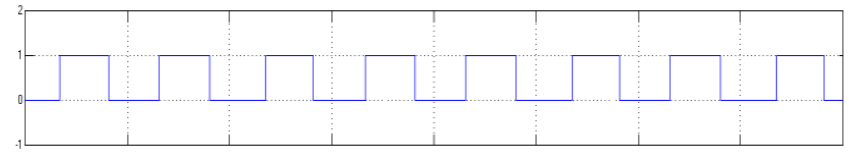

(a) single phase short circult to ground

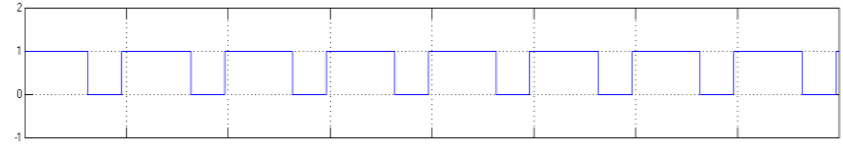

(b) two-phase short circuit to ground occurs

Figure 5. Output After Wave Adjustment of Observer to $U_{d}$

By analyzing, the duty cycle of the wave after adjustment of observer when single phase and two-phase short circuit to ground is 0.480 and 0.662 , and the value remains stable. Thus, after excluding the DC line fault, as long as the duty cycles of rectangular wave of observer, filter, and trigger are respectively calculated, the type of three-phase fault can easily identified, which can optimize the original method.

\section{Conclusion}

This study introduces the features of HVDC Light technologies, and analyzes the related fault diagnosis methods and their advantages and disadvantages, and finally explores the observer-based fault diagnosis method for HVDC system.

The fault observer is designed according to the HVDC system model. The system output is simulated which shows that the output of observer can well reflect the failure occurrence. 
When failure occurs, the changes of DC voltage and current are different. For the observer, different failures correspond to different output curves, the fault can be identified in characteristics based on the integration of the output $U_{d}$ and $I_{d}$ of observer.

Finally, the output waveform is adjusted by Schmitt trigger to obtain optimization plan of regular rectangular wave. The eexperimental results show that the duty cycle of the rectangular wave of fault output by trigger is very stable and unique which can be seen as good evidence for identifying failure types. It should be noted that this method is not used in practice, then, its stability and feasibility still need further exploration in future research.

\section{References}

[1] J. Wen, Y. G. Zhang, M. X. Han and X. N. Xiao, "HDVC Based on Voltage Source Converter - A New Generation of HVDC Technique”, Power System Technology, vol. 27, no. 1, (2003), pp. 47-51.

[2] Y. J. Li, Y. Q. Zhou and Q. Song, "The Development and Application of HVDC Light Technology", High Voltage Engineering, vol. 29, no. 10, (2003), pp. 26-28.

[3] Q. R. Qi, L. W. Jiao, Z. Yan, Y. X. Ni, S. S. Chen and F. L. Wu, "Modeling and Simulation of HVDC with Dynamic Phasors", Proceedings of the Chinese Society for Electrical Engineering, vol. 23, no. 12, (2003), pp. 28-32.

[4] G. G. Yan, T. Chen, G. Mu, W. H. Liu, J. H. Li and Y. F. Huang, 2007, "Dynamic Modeling and NonlinearDecouple Control of HVDC Light System”, Power System Technology, vol. 31, no. 6, (2003), pp. 45-50.

[5] X. Y. Li, "High Voltage Direct Current Transmission System", Science Press, Beijing, China, (2010).

[6] Z. Z. Han, D. J. Pan and Z. J. Zhong, "Observability and Observers of Nonlinear Systems", Control Theory and Applications, vol. 7, no. 4, (1990), pp. 1-9.

[7] B. Liu and W. S. Liu, 2006. "Modern Control Theory", China Machine Press, Beijing, China, (2010).

[8] B. Y. Yan, X. M. Liu, Z. H. Tian, S. J. Shi and F. Yu, "Application of Consensus Filter and SVM in Fault Diagnosis for HVDC Systems", Proceedings of the Chinese Society for Electrical Engineering, vol. 28, no. 1, (2008), pp. 23-29.

[9] Cigre Working Group, "The CIGRE HVDC Benchmark Model - A New Proposal with Revised Parameters", Electra, vol. 30, no. 157, (1994), pp. 61-64.

[10] M. O. Faruque, Y. Y. Zhang and V. Dinavah, "Detailed modeling of CIGRE HVDC benchmark system using PSCAD/EMTDC and PSB/SIMULINK”, IEEE Transactions on Power Delivery, vol. 21, no. 1, (2006), pp. 378-387

[11] Q. Yu and N. Cao, "Modeling and Simulation of Power System Based on MATLAB/Simulink", China Machine Press, Beijing, China, (2011).

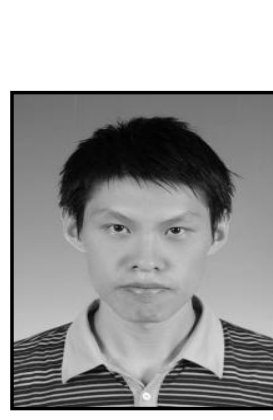

\section{Author}

Yiming Jiang received his B.S. degree in measurement technology and instrumentation from East China University of Science and Technology. He currently studies for a master's degree in instrument science and technology at Tianjin University. 